\title{
Numerical Simulation of the Heat Conduction in Composite Materials
}

\author{
R. Čiegis, G. Jankevičiūtè and O. Suboč \\ Vilnius Gediminas Technical University \\ Saulètekio av. 11, LT-10223, Vilnius, Lithuania \\ E-mail(corresp.): rc@vgtu.lt \\ E-mail: Gerda.Jankeviciute@vgtu.1t;os@vgtu.lt
}

Received December 4, 2009; revised January 18, 2010; published online February 15, 2010

\begin{abstract}
In this paper we develop and validate mathematical models and numerical algorithms for the heat transfer simulation in composite materials. The main features of the problem deal with the dependence of the heat source on the solution, discontinuous diffusion coefficients and nonlinear convection and radiation boundary conditions. The differential problem is approximated by the finite volume discrete scheme. It is proved that for a sufficiently small parameter, which defines the dependence of the source term on the solution, the discrete problem has a unique solution which converges to the solution of the differential problem. Linearization of the nonlinear problem is done by using the Picard method and the convergence of the iterations is proved. Results of numerical experiments are presented.
\end{abstract}

Keywords: heat conduction, nonlinear boundary conditions, finite volume method, convergence analysis, Picard's iterations.

AMS Subject Classification: 65 N20.

\section{Introduction}

The main purpose of this paper is to study a finite volume approximation to the solution of the steady-state heat radiation with nonlinear Stefan-Boltzmann boundary condition in two dimensional rectangular region. The domain is made up of three layers of different materials with different thermal diffusion coefficients. We note, that one material is metal, the other two are isolators, therefore the diffusion coefficients have very different values. The heat source is generated by a current in the metal region and it depends linearly on the temperature of the body.

The elliptic nonlinear equations with nonlinear boundary conditions arise as mathematical models of many interesting processes in physics, technology, engineering. Here we mention applications in engineering for detection the 
corrosion of containers, where direct and inverse heat transfer problems in composite materials are solved $[2,9,18]$, and optimal design of electrical cables $[5,6,11]$.

The paper is organized as follows. In Section 2, we give a brief description of the model, describe the main processes of heat conduction in composite structures. A discrete scheme is constructed using the Finite Volume Method (FVM). A special attention is given to the approximation of the nonlinear Robin boundary conditions. The stability and convergence of the 1D difference scheme is presented in Section 3. The Picard iterative method is used to linearize the nonlinear discrete problem. The convergence of iterations is also proved in Section 3. Some details of implementation of the 2D discrete scheme and results of computational experiments are given in Section 4 .

\section{Problem Formulation}

In domain $D=\left(0, L_{1}\right) \times\left(0, L_{2}\right)$ we solve the nonlinear stationary problem, which describes a distribution of the temperature $T(X, t)$ in the domain $D$, which describes a composite structure of metal and isolator materials (see, Figure 1).

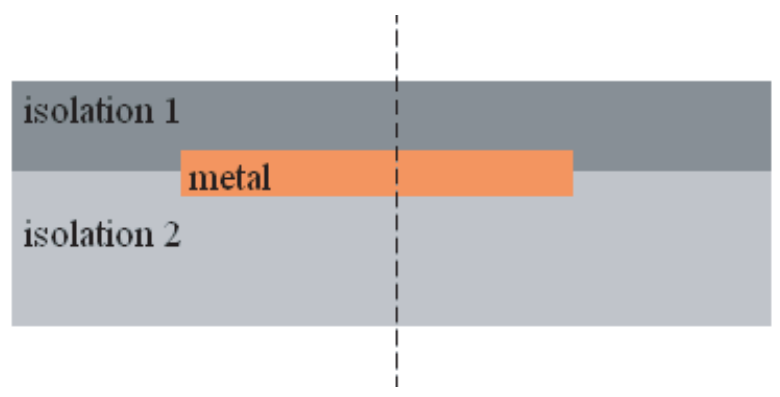

Figure 1. Scheme of the composite structure of metal and isolator materials.

\subsection{Mathematical model of heat conduction in composite structure}

The mathematical model consists of the elliptic differential equation [10]:

$$
-\sum_{i=1}^{2} \frac{\partial}{\partial x_{i}}\left(k(X) \frac{\partial T}{\partial x_{i}}\right)=\left(\frac{I(X)}{A}\right)^{2} \rho_{0}(1+c(T-20)), \quad X \in D,
$$

subject to the symmetry condition on $\partial D_{1}=\left\{\left(0, x_{2}\right), x_{2} \in\left[0, L_{2}\right]\right\}$

$$
k(X) \frac{\partial T}{\partial x_{1}}=0, \quad X \in \partial D_{1}
$$

and the nonlinear boundary condition on the remaining part of the boundary:

$$
k(X) \frac{\partial T}{\partial \eta}+\alpha_{K}(T)\left(T(X, t)-T_{a}\right)+\varepsilon \sigma\left(\tilde{T}^{4}-\tilde{T}_{a}^{4}\right)=0, \quad X \in \partial D \backslash \partial D_{1},
$$


where $\tilde{T}=T+273.15$ is a temperature in the Kelvin scale. This boundary condition describes the heat transfer on the boundary due to the Newton convection and the Stefan-Boltzmann radiation processes. The convection heat transfer coefficient $\alpha_{K}(T) \geq \alpha_{0}>0$ for $T \geq T_{a}$.

Here $I$ is a given current, $A$ is an area of the metal, $\rho_{0}$ is the specific resistivity of the conductor, $c>0$ is a constant, $T_{a}$ is the temperature of the environment. Let us define the metal domain

$$
D_{M}=\left\{X \in D: \quad 0<x_{1}<L_{1 M}<L_{1}, \quad 0<l_{2 M}<x_{2}<L_{2 M}<L_{2}\right\} .
$$

Then the current function $I(X)$ and diffusion coefficient $k(X)$ have the following form

$$
I(X)=\left\{\begin{array}{ll}
I_{0} \quad \text { if } X \in \bar{D}_{M}, \\
0 \quad \text { if } X \in D \backslash \bar{D}_{M},
\end{array} \quad k(X)= \begin{cases}K_{M} & \text { if } X \in \bar{D}_{M}, \\
K_{I} & \text { if } X \in D \backslash \bar{D}_{M},\end{cases}\right.
$$

and $0<K_{I} \ll K_{M}$. The standard continuity conditions are specified at the points of discontinuity of coefficients

$$
[T(x, t)]=0, \quad\left[k \frac{\partial T}{\partial n}\right]=0, \quad X \in \partial D_{M},
$$

where $n$ is a normal vector.

\subsection{Discrete approximation}

Let us introduce in the domain $D$ a non-uniform grid $D_{h}=\omega_{1 h} \times \omega_{2 h}$, where

$$
\begin{aligned}
& \omega_{1 h}=\left\{x_{1 i}: \quad x_{1 i}=x_{1, i-1}+h_{1, i-0.5}, \quad i=1, \ldots, N_{1}-1\right\}, \quad x_{1, N_{1}}=L_{1}, \\
& \omega_{2 h}=\left\{x_{2 j}: \quad x_{2 j}=x_{2, j-1}+h_{2, j-0.5}, \quad j=1, \ldots, N_{2}-1\right\}, \quad x_{2, N_{2}}=L_{2} \text {. }
\end{aligned}
$$

For grid steps we also use notation $h_{1, i}=0.5\left(h_{1, i-0.5}+h_{1, i+0.5}\right), h_{2, j}=$ $0.5\left(h_{2, j-0.5}+h_{2, j+0.5}\right)$.

Let us define a set of discrete functions $U \in H$, where $U_{i j}:=U\left(x_{1 i}, x_{2 j}\right)$ denotes a value of function $U$ at discrete point $X_{i j} \in \bar{D}_{h}$. We also define the following difference operators:

$$
\begin{aligned}
& V_{i+0.5, j}:=\partial_{x_{1}} U_{i j}=\frac{U_{i+1, j}-U_{i j}}{h_{1, i+0.5}}, \quad W_{i j}:=\partial_{\bar{x}_{1}} V_{i+0.5, j}=\frac{V_{i+0.5, j}-V_{i-0.5, j}}{h_{1, i}}, \\
& V_{i, j+0.5}:=\partial_{x_{2}} U_{i j}=\frac{U_{i, j+1}-U_{i j}}{h_{2, j+0.5}}, \quad W_{i j}:=\partial_{\bar{x}_{2}} V_{i, j+0.5}=\frac{V_{i, j+0.5}-V_{i, j-0.5}}{h_{2, j}} .
\end{aligned}
$$

Integrating the differential equation (2.1) over the control volume and approximating the obtained integrals with an individual quadrature for each term, the differential problem is approximated by the conservative scheme

$$
-\sum_{\alpha=1}^{2} \partial_{\bar{x}_{\alpha}}\left(k\left(X_{i j, \alpha}\right) \partial_{x_{\alpha}} U_{i j}\right)=\left(\frac{I_{i j}}{A}\right)^{2} \rho_{0}\left(1+c\left(U_{i j}-20\right)\right), \quad X_{i j} \in D_{h},
$$


where the notation $X_{i j, \alpha}=\left(x_{1, i+\frac{1}{2} \delta_{1 \alpha}}, x_{2, j+\frac{1}{2} \delta_{2 \alpha}}\right)$ is used, here $\delta_{i j}$ is the Kroneker symbol. The boundary conditions are approximated in a similar way. The symmetry condition (2.2) is approximated by:

$$
\begin{aligned}
-\frac{2}{h_{1,0.5}} k\left(X_{0 j, 1}\right) \partial_{x_{1}} U_{0 j} & -\partial_{\bar{x}_{2}}\left(k\left(X_{0 j, 2}\right) \partial_{x_{2}} U_{0 j}\right) \\
& =\left(\frac{I_{0 j}}{A}\right)^{2} \rho_{0}\left(1+c\left(U_{0 j}-20\right)\right), \quad x_{2 j} \in \omega_{2 h} .
\end{aligned}
$$

We restrict to presentation of discrete boundary equations only on one line of the boundary. The boundary conditions (2.3) are approximated by

$$
\begin{aligned}
-\frac{2}{h_{2,0.5}} k\left(X_{i 0,2}\right) \partial_{x_{2}} U_{i 0} & -\partial_{\bar{x}_{1}}\left(k\left(X_{i 0,1}\right) \partial_{x_{1}} U_{i 0}\right) \\
& +\frac{2}{h_{2,0.5}} \alpha_{G}\left(U_{i 0}\right)\left(U_{i 0}-T_{a}\right)=0, \quad x_{1 i} \in \omega_{1 h},
\end{aligned}
$$

where $\alpha_{G}(U)=\alpha_{K}(U)+\varepsilon \sigma\left(\tilde{U}^{2}+\tilde{T}_{a}^{2}\right)\left(\tilde{U}+\tilde{T}_{a}\right)$.

It remains to write discrete approximations at the corner points of the domain $D_{h}$. Let us consider this equation for the left upper corner:

$$
\begin{aligned}
-\frac{2}{h_{1,0.5}} k\left(X_{0, N_{2}, 1}\right) \partial_{x_{1}} U_{0, N_{2}} & +\frac{2}{h_{2, N_{2}-0.5}}\left(k\left(X_{i, N_{2}-1,2}\right) \partial_{x_{2}} U_{i, N_{2}-1}\right. \\
& \left.+\alpha_{G}\left(U_{i, N_{2}}\right)\left(U_{i, N_{2}}-T_{a}\right)\right)=0
\end{aligned}
$$

Taking into account (2.4) we can write the discrete scheme in an operator form:

$$
L_{h}(U) U_{i j}=\left(\frac{I_{i j}}{A}\right)^{2} \rho_{0}\left(1+c\left(U_{i j}-20\right)\right), \quad X_{i j} \in \bar{D}_{h},
$$

where the nonlinearity in $L_{h}(U)$ is due to nonlinear function $\alpha_{G}(U)$.

Finite volume approximations of second order elliptic equations with discontinuous coefficients where considered in $[8,16]$ (see also references given therein). The existence and convergence of finite difference schemes for elliptic problems with nonlinear boundary coefficients and nonlinear $f$ was considered in many papers. First results were given in [3], where a variational formulation of $1 \mathrm{D}$ problem was used and approximation by splines was applied. A good review on numerical algorithms for nonlinear $3 \mathrm{D}$ heat radiation problem and existence and convergence analysis of the Galerkin method is presented in [12]. Their analysis is based on minimization of the obtained functional of potential energy. Moreover, they apply the Newton iterative method for solving the nonlinear equations resulting from the minimization problem and prove the standard convergence result for a sufficiently good initial approximation.

\section{Analysis of 1D Discrete Algorithm}

In order to simplify all notation we restrict to one dimensional problem and solve problem in domain where $D=\left\{x \in D: 0<x<L_{1}\right\}$ and the metal 
domain is defined as $D_{M}=\left\{x \in D: 0<l_{1} \leq x \leq l_{2}<L_{1}\right\}$. Then we get the discrete problem

$$
L_{h}(U) U_{i}=F\left(x_{i}, U_{i}\right), \quad x_{i} \in \bar{D}_{h},
$$

where the operator $L_{h}(V)$ and vector-function $F$ are defined as

$$
\begin{aligned}
L_{h}(V) U=\left\{\begin{array}{l}
l l-\frac{2}{h_{1 / 2}}\left(k\left(x_{1 / 2}\right) \partial_{x} U_{0}-\alpha_{G}\left(V_{0}\right) U_{0}\right), \quad i=0, \\
-\partial_{\bar{x}}\left(k\left(x_{i+1 / 2}\right) \partial_{x} U_{i}\right), \quad 0<i<N, \\
\frac{2}{h_{N-1 / 2}}\left(k\left(x_{N-1 / 2}\right) \partial_{x} U_{N-1}+\alpha_{G}\left(V_{N}\right) U_{N}\right), \quad i=N,
\end{array}\right. \\
F(X, U)=\left\{\begin{array}{l}
\frac{2}{h_{1 / 2}} \alpha_{G}\left(U_{0}\right) T_{a}, \quad i=0, \\
\left(\frac{I_{i}}{A}\right)^{2} \rho_{0}\left(1+c\left(U_{i}-20\right)\right), \quad 1 \leq i<N, \\
\frac{2}{h_{1 / 2}} \alpha_{G}\left(U_{N}\right) T_{a}, \quad i=N .
\end{array}\right.
\end{aligned}
$$

\subsection{Existence and uniqueness of a solution}

First we will show, that if a solution of problem (3.1) exists, then $U_{i} \geq T_{a}$, $x_{i} \in \bar{D}_{h}$. Let us assume that the following inequality is valid for a density of heat source:

$$
1+c\left(T_{a}-20\right) \geq 0 .
$$

Lemma 1. Assume that hypothesis (3.2) is satisfied and $\alpha_{K}(V) \geq 0$. Then for a solution $U$ of (3.1), if it exists, we have that

$$
U_{i} \geq T_{a} \quad x_{i} \in \bar{D}_{h} .
$$

Proof. Consider two discrete linear problems for $W^{j}, j=1,2$ :

$$
\left\{\begin{array}{l}
-k\left(x_{1 / 2}\right) \partial_{x} W_{0}^{j}+\alpha_{0}^{j}\left(W_{0}^{j}-T_{a}\right)=0, \quad i=0, \\
-\partial_{\bar{x}}\left(k\left(x_{i+1 / 2}\right) \partial_{x} W_{i}^{j}\right)=f_{i}^{j}, \quad 0<i<N, \\
k\left(x_{N-1 / 2}\right) \partial_{x} W_{N-1}^{j}+\alpha_{N}^{j}\left(W_{N}^{j}-T_{a}\right)=0, \quad i=N .
\end{array}\right.
$$

where $\alpha_{l}^{1} \geq \alpha_{l}^{2} \geq 0, l=0, N$, and $f_{i}^{2} \geq f_{i}^{1} \geq 0, x_{i} \in D_{h}$. Due to these assumptions and since matrix of the system is an M-matrix [15], we have that $W_{i}^{j} \geq T_{a}$ for $x_{i} \in \bar{D}_{h}$. Subtracting all equations of the first problem from the second problem, we get for $V=W^{2}-W^{1}$ :

$$
\left\{\begin{array}{l}
-k\left(x_{1 / 2}\right) \partial_{x} V_{0}+\alpha^{2} V_{0}=\left(\alpha_{0}^{1}-\alpha_{0}^{2}\right)\left(W_{0}^{1}-T_{a}\right), \quad i=0, \\
-\partial_{\bar{x}}\left(k\left(x_{i+1 / 2}\right) \partial_{x} V_{i}^{j}\right)=f_{i}^{2}-f_{i}^{1}, \quad 0<i<N, \\
k\left(x_{N-1 / 2}\right) \partial_{x} W_{N-1}^{j}+\alpha^{2} V_{N}=\left(\alpha_{N}^{1}-\alpha_{N}^{2}\right)\left(W_{N}^{1}-T_{a}\right), \quad i=N .
\end{array}\right.
$$

Since $W_{i}^{1} \geq T_{a}, f_{i}^{2}-f_{i}^{1} \geq 0, \alpha_{l}^{1}-\alpha_{l}^{2}>0$, it follows from the maximum principle that $V_{i} \geq 0$. This implies that $W^{2} \geq W^{1}$. Thus a bound from below 
$U \geq W$ for a solution $U$ of problem (3.1) is obtained by solving a problem with the maximal value of the convection coefficient (i.e., $\alpha=\infty$ ) and the minimal source density (i.e., $f \equiv 0$, see estimate $(3.2))$ :

$$
\left\{\begin{array}{l}
W_{0}=T_{a}, \quad i=0 \\
-\partial_{\bar{x}}\left(k\left(x_{i+1 / 2}\right) \partial_{x} W_{i}\right)=0, \quad 0<i<N \\
W_{N}=T_{a}, \quad i=N
\end{array}\right.
$$

From the maximum principle it follows that $W \geq T_{a}$, which completes the proof.

For notational convenience, we set $V:=U-T_{a}$, where $V$ satisfies the following nonlinear discrete problem

$$
\left\{\begin{array}{l}
-k\left(x_{1 / 2}\right) \partial_{x} V_{0}+\tilde{\alpha}_{K}\left(V_{0}\right) V_{0}+\varepsilon \sigma\left(\left(V_{0}+\tilde{T}_{a}\right)^{4}-\tilde{T}_{a}^{4}\right)=0, \quad i=0, \\
-\partial_{\bar{x}}\left(k\left(x_{i+1 / 2}\right) \partial_{x} V_{i}\right)=\left(\frac{I_{i}}{A}\right)^{2} \rho_{0}\left(1+c\left(V_{i}^{j}+T_{a}-20\right)\right), \quad 0<i<N, \\
k\left(x_{N-\frac{1}{2}}\right) \partial_{x} V_{N-1}+\tilde{\alpha}_{K}\left(V_{N}\right) V_{N}+\varepsilon \sigma\left(\left(V_{N}+\tilde{T}_{a}\right)^{4}-\tilde{T}_{a}^{4}\right)=0, \quad i=N,
\end{array}\right.
$$

where $\tilde{\alpha}_{K}(V):=\alpha_{K}\left(V+T_{a}\right)$.

In the following analysis we will use the relation between finite volume scheme and the minimization problem (for a similar analysis of Galerkin type approximations see $[3,9,12]$, linear finite difference schemes [16]). We define a bilinear form

$$
a(Z, W):=\sum_{i=0}^{N-1} k\left(x_{i+1 / 2}\right)\left(\partial_{x} Z_{i}\right)\left(\partial_{x} W_{i}\right) h_{i+1 / 2}-\sum_{i=1}^{N-1} c f_{i} Z_{i} W_{i} h_{i}, Z, W \in H\left(D_{h}\right),
$$

where $f_{i}:=\left(I_{i} / A\right)^{2} \rho_{0}$, and a linear functional

$$
R(Z):=\sum_{i=1}^{N-1} f_{i} Z_{i} h_{i}+\varepsilon \sigma \tilde{T}_{a}^{4}\left(Z_{0}+Z_{N}\right), \quad Z \in H\left(D_{h}\right) .
$$

Let introduce notation $A(u):=\int_{0}^{u} \alpha_{K}\left(s+T_{a}\right) s d s$.

Consider the functional of energy

$$
J(Z)=\frac{1}{2} a(Z, Z)+A\left(Z_{0}\right)+A\left(Z_{n}\right)+\frac{\varepsilon \sigma}{5}\left(\left(Z_{0}+\tilde{T}_{a}\right)^{5}+\left(Z_{N}+\tilde{T}_{a}\right)^{5}\right)-F(Z) .
$$

Let us define a set of non-negative functions

$$
B=\left\{Z \in H\left(D_{h}\right): Z_{i} \geq 0, \quad x_{i} \in \bar{D}_{h}\right\} .
$$

Next we formulate the following minimization problem: find $V \in B$ such that

$$
J(V)=\inf _{Z \in B} J(Z) .
$$


Remark 1. The solution of variational problem (3.3) is equivalent to the solution of discrete problem (3.1). Indeed, the solution of (3.3) is characterized by the condition

$$
J^{\prime}(V ; Z)=0 \quad \forall Z \in B,
$$

where the first Gateaux derivative of $J$ is defined as

$$
J^{\prime}(V ; Z):=\lim _{t \rightarrow 0} \frac{J(V+t Z)-J(V)}{t}, \quad V, Z \in B .
$$

Simple computations give that

$$
\begin{aligned}
J^{\prime}(V ; Z)=a(V, Z) & +\left(\tilde{\alpha}_{K}\left(V_{0}\right) V_{0}+\varepsilon \sigma\left(V_{0}+\tilde{T}_{a}\right)^{4}\right) Z_{0} \\
& +\left(\tilde{\alpha}_{K}\left(V_{N}\right) V_{N}+\varepsilon \sigma\left(V_{N}+\tilde{T}_{a}\right)^{4}\right) Z_{N}-R(Z),
\end{aligned}
$$

or after summation by parts in the bilinear form, we get

$$
\sum_{i=0}^{N}\left(L_{h}\left(V_{i}\right) V_{i}-F\left(x_{i}, V_{i}\right)\right) Z_{i}=0, \quad \forall Z \in B
$$

which coincides with discrete problem (3.1).

Theorem 1. Assume that hypothesis (3.2) is satisfied and

$$
\alpha_{K}\left(T_{a}\right)=\alpha_{0}>0, \quad \alpha_{K}^{\prime}(v) \geq 0, \quad \forall v \geq T_{a},
$$

Then the functional $J$ is continuous on B. For a sufficiently small $c \leq c_{0}$ this functional is also strictly convex and coercive on $B$. Therefore, the minimization problem (3.4) has a unique solution $V \in B$.

Proof. It is easy to check by direct computations that $J$ is continuous on $B$. The second Gateaux derivative of $J$ is given as

$$
\begin{aligned}
J^{\prime \prime}(V ; Z, W)=a(W, Z) & +\left(\tilde{\alpha}_{K}\left(V_{0}\right)+\tilde{\alpha}_{K}^{\prime}\left(V_{0}\right) V_{0}+4 \varepsilon \sigma\left(V_{0}+\tilde{T}_{a}\right)^{3}\right) W_{0} Z_{0} \\
& +\left(\tilde{\alpha}_{K}\left(V_{N}\right)+\tilde{\alpha}_{K}^{\prime}\left(V_{N}\right) V_{N}+4 \varepsilon \sigma\left(V_{N}+\tilde{T}_{a}\right)^{3}\right) W_{N} Z_{N} .
\end{aligned}
$$

Let us define the norm $\|\cdot\|_{B}$ in the space B by

$$
\|U\|_{B}^{2}:=\sum_{i=0}^{N-1} k\left(x_{i+1 / 2}\right)\left(\partial_{x} U_{i}\right)^{2} h_{i+1 / 2}+\left(\alpha_{0}+4 \varepsilon \sigma \tilde{T}_{a}^{3}\right)\left(U_{0}^{2}+U_{N}^{2}\right) .
$$

It follows from the well known embedding theorem [16], that there exists a constant $\gamma>0$ such that

$$
\inf _{Z \in H} \frac{\|Z\|_{B}^{2}}{\sum_{i=1}^{N-1} f_{i} Z_{i}^{2} h_{i}} \geq \frac{1}{\gamma}
$$

Let us take $c_{0}$ such that $1-c_{0} \gamma \geq 1 / 2$. Then for $c \leq c_{0}$ and any $V, Z \in B$ we have

$$
J^{\prime \prime}(V ; Z, Z) \geq \frac{1}{2}\|Z\|_{B}^{2} \geq 0
$$


It is easy to check that $J^{\prime \prime}(V ; Z, Z)=0$ if and only if $Z \equiv 0$ for any $V \in B$. We have proved that $J$ is strictly convex on $B$.

Let us assume that $f_{i} \leq M_{f}, x_{i} \in D_{h}$. Since $\tilde{T}_{a}>4$, then applying similar analysis we get the estimate

$$
J(V) \geq \frac{1}{2}\|V\|_{B}^{2}-\sqrt{\gamma M_{f} L}\|V\|_{B},
$$

which implies that $J(V) \rightarrow \infty$ as $\|V\|_{B} \rightarrow \infty$, thus $J$ is coercive on $B$. From all these properties of $J$ it follows that minimization problem (3.4) has a unique solution $V \in B$ (see, [7]).

Remark 2. The existence of a unique solution of 2D discrete problem (2.5) can be proved analogically.

\subsection{Convergence analysis}

Let us denote the error function of the discrete solution of (3.1) as $Z_{i}=U_{i}$ $u\left(x_{i}\right), x_{i} \in \bar{D}_{h}$. By putting it into the finite-difference scheme we get a discrete problem for the error functions:

$$
\left\{\begin{array}{l}
-k\left(x_{1 / 2}\right) \partial_{x} Z_{0}+\left(\alpha_{K}\left(U_{0}\right)+\alpha_{K}^{\prime}\left(U_{0}^{\theta}\right)+\varepsilon \sigma \tilde{P}_{0}\right) Z_{0}=\eta_{1 / 2}, \quad i=0, \\
-\partial_{\bar{x}}\left(k\left(x_{i+1 / 2}\right) \partial_{x} Z_{i}\right)=\left(\frac{I_{i}}{A}\right)^{2} \rho_{0} c Z_{i}+\partial_{\bar{x}} \eta_{i+1 / 2}, \quad 0<i<N, \\
k\left(x_{N-\frac{1}{2}}\right) \partial_{x} Z_{N-1}+\left(\alpha_{K}\left(U_{N}\right)+\alpha_{K}^{\prime}\left(U_{N}^{\theta}\right)+\varepsilon \sigma \tilde{P}_{N}\right) Z_{N}=\eta_{N-\frac{1}{2}}, \quad i=N,
\end{array}\right.
$$

here we use notation:

$$
U_{j}^{\theta}:=\theta U_{j}+(1-\theta) u_{j}, \quad 0<\theta<1, \quad \tilde{P}_{j}=\tilde{U}_{j}^{3}+\tilde{U}_{j}^{2} \tilde{u}_{j}+\tilde{U}_{j} \tilde{u}_{j}^{2}+\tilde{u}_{j}^{3} .
$$

Functions $\eta_{j+1 / 2}, j=0, \ldots, N-1$, define the truncation errors in approximation of fluxes

$$
\eta_{j+1 / 2}:=k\left(x_{j+1 / 2}\right) \partial_{x} u_{j},
$$

and they can be estimated in a standard way as $\eta_{j+1 / 2}=C h_{j+1 / 2}^{2}$.

Theorem 2. Assume that assumptions (3.2), (3.5) are satisfied. Then for a sufficiently small $c \leq c_{0}$ the solution of discrete problem (3.1) converges to the solution of $1 D$ differential problem and the following estimate is valid

$$
\|U-u\|_{B} \leq C h^{2}, \quad h=\max _{j=1, \ldots, N} h_{j-1} .
$$

Proof. We multiply each equation of (3.7) by $Z_{i} h_{i}$, add the obtained equalities and apply the formula of summation by parts, then we get the equality

$$
\begin{aligned}
a(Z, Z) & +\left(\alpha_{K}\left(U_{0}\right)+\alpha_{K}^{\prime}\left(U_{0}^{\theta}\right)+\varepsilon \sigma \tilde{P}_{0}\right) Z_{0}^{2}+\left(\alpha_{K}\left(U_{N}\right)\right. \\
& \left.+\alpha_{K}^{\prime}\left(U_{N}^{\theta}\right)+\varepsilon \sigma \tilde{P}_{N}\right) Z_{N}^{2}=\sum_{i=1}^{N-1} \partial_{\bar{x}} \eta_{i+1 / 2} Z_{i} h_{i}+\eta_{1 / 2} Z_{0}+\eta_{N-1 / 2} Z_{N} .
\end{aligned}
$$


Recalling that $U, u \geq T_{a}$, using the embedding estimate (3.6) on the left side of this inequality and taking a sufficiently small $c \leq c_{0}$, we get that

$$
\begin{aligned}
\frac{1}{2}\|Z\|_{B}^{2} \leq a(Z, Z) & +\left(\alpha_{K}\left(U_{0}\right)+\alpha_{K}^{\prime}\left(U_{0}^{\theta}\right)+\varepsilon \sigma \tilde{P}_{0}\right) Z_{0}^{2} \\
& +\left(\alpha_{K}\left(U_{N}\right)+\alpha_{K}^{\prime}\left(U_{N}^{\theta}\right)+\varepsilon \sigma \tilde{P}_{N}\right) Z_{N}^{2} .
\end{aligned}
$$

Using the formula of summation by parts and the $\varepsilon$ inequality

$$
|a b| \leq \varepsilon a^{2}+\frac{1}{4 \varepsilon} b^{2},
$$

we have

$$
\begin{aligned}
& \sum_{i=1}^{N-1} \partial_{\bar{x}} \eta_{i+1 / 2} Z_{i} h_{i}+\eta_{1 / 2} Z_{0}+\eta_{N-1 / 2} Z_{N}=-\sum_{i=0}^{N-1} \eta_{i+1 / 2} \partial_{x} Z_{i} h_{i+1 / 2} \\
& \quad+2 \eta_{N-1 / 2} Z_{N} \leq \frac{1}{4}\|Z\|_{B}^{2}+\sum_{i=0}^{N-1} \frac{\eta_{i+1 / 2}^{2}}{k_{i+1 / 2}} h_{i+1 / 2}+\frac{\eta_{N-1 / 2}^{2}}{\alpha_{0}+4 \varepsilon \sigma}
\end{aligned}
$$

Combining both estimates we obtain that

$$
\|Z\|_{B} \leq C \max _{0 \leq j<N}\left|\eta_{j+1 / 2}\right|,
$$

which completes the proof.

\subsection{Convergence analysis of Picard's iterations}

The fixed point iteration is a very convenient method, since we have a possibility to control the properties of the system of linear equations obtained after a linearization step. Choosing an initial guess $U^{0} \in B$, we find a solution of (2.5) or (3.9) by the Picard iterative algorithm:

$$
L_{h}\left(U^{s-1}\right) U^{s}=F\left(X, U^{s-1}\right), \quad s=1,2, \ldots, S .
$$

\subsubsection{Positivity of iterations}

We take the initial approximation as $U_{i}^{0}=T_{a}, x_{i} \in \bar{D}_{h}$.

Lemma 2. Assume that hypothesis (3.2) is satisfied and $U_{i}^{0}=T_{a}, x_{i} \in \bar{D}_{h}$. Then for sequence $\left\{U^{s}\right\}$ generated by the iterative algorithm (3.9) we have that

$$
U_{i}^{s} \geq T_{a} \quad x_{i} \in \bar{D}_{h}, \quad \forall s \geq 1 .
$$

Proof. For $U^{0}$ estimate (3.10) is valid. Let assume that $U^{s-1} \geq T_{a}$. Then, taking in the proof of Lemma 1 coefficients $\alpha^{1}=\infty, \alpha_{l}^{2}=\alpha_{G}\left(U_{l}^{s-1}\right), l=0, N$, $f^{1} \equiv 0, f^{2}=F\left(X, U^{s-1}\right) \geq 0$ we obtain that $U^{s} \geq T_{a}$. The proof is completed by using the mathematical induction method.

Remark 3. If a more strict condition $1+\alpha_{\rho}\left(T_{a}-20\right)>0$ is satisfied, then we have that $U_{i}^{s}>T_{a}, x_{i} \in \bar{D}_{h}, \forall s \geq 1$. 


\subsubsection{Convergence of iterations}

First, we consider the case when $\alpha_{\rho}=0$, i.e. the heat source density does not depend on the solution. Then it follows from Remark 3 that $U_{i}^{s}>T_{a}$ for all $s \geq 1$.

Lemma 3. Assume that $\alpha_{G}^{\prime}(v)>0$ for $v>T_{a}$ and $U_{i}^{0}=T_{a}, x_{i} \in \bar{D}_{h}$. Then for sequence $\left\{U^{s}\right\}$ generated by the iterative algorithm (3.9) we have the following estimates

$$
U_{i}^{0}<U_{i}^{2}<\ldots<U_{i}^{2 j} \leq U_{i} \leq U_{i}^{2 j+1}<\ldots<U_{i}^{3}<U_{i}^{1}, \quad x_{i} \in \bar{D}_{h} .
$$

Proof. Let us take discrete functions $T_{a}<V_{i}^{1}<V_{i}^{2}, x_{i} \in \bar{D}_{h}$ and solve problems

$$
L_{h}\left(V^{j}\right) W^{j}=F\left(X, V^{j}\right), \quad j=1,2 .
$$

Then it follows from the proof of Lemma 1, that

$$
W_{i}^{2}<W_{i}^{1}, \quad x_{i} \in \bar{D}_{h} .
$$

Then it follows from Remark 3 that $U_{i}^{1}>T_{a}=U_{i}^{0}$. Using (3.12) we get that $U_{i}^{2}<U_{i}^{1}$ and also $U_{i}^{2}>T_{a}=U_{i}^{0}$. Repeating these iterations we prove (3.11).

This lemma gives very useful error bounds of iterations $U^{s}$ but this result is not sufficient to prove the convergence of Picard's iterations.

Theorem 3. Assume that assumptions (3.2), (3.5) and

$$
\alpha_{G}^{\prime}(U)\left(U-T_{a}\right) \leq q \alpha_{G}(U), \quad q<1
$$

are satisfied. Then for a sufficiently small $c \leq c_{0}$ iterations (3.9) converge to the solution of discrete problem (3.1) and the following estimate is valid

$$
\begin{aligned}
& (1-\mu)\left\|Z^{s}\right\|_{B}^{2}+\mu\left(\alpha_{G}\left(U_{0}\right)\left(Z_{0}^{s}\right)^{2}+\alpha_{G}\left(U_{N}\right)\left(Z_{N}^{s}\right)^{2}\right) \leq(q+c \gamma) \\
& \quad \times\left[(1-\mu)\left\|Z^{s-1}\right\|_{B}^{2}+\mu\left(\alpha_{G}\left(U_{0}\right)\left(Z_{0}^{s-1}\right)^{2}+\alpha_{G}\left(U_{N}\right)\left(Z_{N}^{s-1}\right)^{2}\right)\right],
\end{aligned}
$$

where $Z^{s}=U^{s}-U$ is the error of iterations, and $\mu=q /(q+c \gamma)$.

Proof. Error function $Z^{s}$ satisfies the boundary value problem

$$
\left\{\begin{array}{l}
-k\left(x_{1 / 2}\right) \partial_{x} Z_{0}^{s}+\alpha_{G}\left(U_{0}^{s-1}\right) Z_{0}^{s}=\alpha_{G}^{\prime}\left(U_{0}^{\theta, s-1}\right)\left(U_{0}-T_{a}\right) Z_{0}^{s-1}, \quad i=0, \\
-\partial_{\bar{x}}\left(k\left(x_{i+1 / 2}\right) \partial_{x} Z_{i}^{s}\right)=\left(\frac{I_{i}}{A}\right)^{2} \rho_{0} c Z_{i}^{s-1}, \quad 0<i<N, \\
k\left(x_{N-\frac{1}{2}}\right) \partial_{x} Z_{N-1}^{s}+\alpha_{G}\left(U_{N}^{s-1}\right) Z_{N}^{s}=\alpha_{G}^{\prime}\left(U_{N}^{\theta, s-1}\right)\left(U_{N}-T_{a}\right) Z_{N}^{s-1}, \quad i=N,
\end{array}\right.
$$

where we set $U^{\theta, s}=\theta U+(1-\theta) U^{s}$. In order to make all technical details more simple, in the following we apply the freezing method and compute nonlinear coefficients at $U$. Otherwise, hypothesis (3.13) should be changed to a variational inequality in a neighbourhood of the solution. We multiply each equation 
by $Z_{i}^{s} h_{i}$, add the obtained equalities and apply the formula of summation by parts, then we get the equality

$$
\begin{gathered}
\sum_{i=0}^{N-1} k_{i+1 / 2}\left(\partial_{x} Z^{s}\right)^{2} h_{i+1 / 2}+\alpha_{G}\left(U_{0}\right)\left(Z_{0}^{s}\right)^{2}+\alpha_{G}\left(U_{N}\right)\left(Z_{N}^{s}\right)^{2}=c \sum_{i=1}^{N-1} f_{i} Z_{i}^{s} Z_{i}^{s-1} h_{i} \\
+\alpha_{G}^{\prime}\left(U_{0}\right)\left(U_{0}-T_{a}\right) Z_{0}^{s} Z_{0}^{s-1}+\alpha_{G}^{\prime}\left(U_{N}\right)\left(U_{N}-T_{a}\right) Z_{N}^{s} Z_{N}^{s-1}
\end{gathered}
$$

Using (3.6), (3.13) and the $\varepsilon$-inequality, it is straightforward to show for $0 \leq$ $\mu \leq 1$ that

$$
\begin{gathered}
(1-\mu)\left\|Z^{s}\right\|_{B}^{2}+\mu\left(\alpha_{G}\left(U_{0}\right)\left(Z_{0}^{s}\right)^{2}+\alpha_{G}\left(U_{N}\right)\left(Z_{N}^{s}\right)^{2}\right) \\
\leq \sum_{i=0}^{N-1} k_{i+1 / 2}\left(\partial_{x} Z^{s}\right)^{2} h_{i+1 / 2}+\alpha_{G}\left(U_{0}\right)\left(Z_{0}^{s}\right)^{2}+\alpha_{G}\left(U_{N}\right)\left(Z_{N}^{s}\right)^{2}, \\
\alpha_{G}^{\prime}\left(U_{j}\right)\left(U_{j}-T_{a}\right) Z_{j}^{s} Z_{j}^{s-1} \leq q \alpha_{G}\left(U_{j}\right)\left|Z_{j}^{s}\right|\left|Z_{j}^{s-1}\right| \\
\leq \frac{\mu}{2} \alpha_{G}\left(U_{j}\right)\left(\left(Z_{j}^{s}\right)^{2}+\frac{q^{2}}{\mu^{2}}\left(Z_{j}^{s-1}\right)^{2}\right), \quad j=0, N \\
\sum_{i=1}^{N-1} f_{i} Z_{i}^{s} Z_{i}^{s-1} h_{i} \leq \varepsilon_{0} \sum_{i=1}^{N-1} f_{i}\left(Z_{i}^{s}\right)^{2} h_{i}+\frac{1}{4 \varepsilon_{0}} \sum_{i=1}^{N-1} f_{i}\left(Z_{i}^{s-1}\right)^{2} h_{i} \\
\leq \varepsilon_{0} \gamma\left\|Z^{s}\right\|_{B}^{2}+\frac{\gamma}{4 \varepsilon_{0}}\left\|Z^{s-1}\right\|_{B}^{2} \leq \frac{1-\mu}{2}\left(\left\|Z^{s}\right\|_{B}^{2}+\frac{(\gamma c)^{2}}{(1-\mu)^{2}}\left\|Z^{s-1}\right\|_{B}^{2}\right) .
\end{gathered}
$$

Thus if we take $\mu=q /(q+c \gamma)$, then we get estimate (3.14). Let us pick $c_{0}$ such that $q+c_{0} \gamma<1$, then the Picard iteration operator defines a contraction provided that $c \leq c_{0}$.

Remark 4. For the application considered in this paper we have the following generalized convection coefficient

$$
\begin{aligned}
& \alpha_{G}(V):=\alpha_{K}(V)+\alpha_{R}(V), \quad \alpha_{K}(V)=\left(c_{1}+c_{2} V^{1 / 6}\right)^{2}, \\
& \alpha_{R}(V)=\varepsilon \rho\left(\tilde{V}^{3}+\tilde{V}^{2} \tilde{T}_{a}+\tilde{V} \tilde{T}_{a}^{2}+\tilde{T}_{a}^{3}\right) .
\end{aligned}
$$

It is easy to see, that

$$
\begin{aligned}
\frac{\alpha_{K}^{\prime}(U)\left(U-T_{a}\right)}{\alpha_{K}(U)} \leq \frac{c_{2} U^{1 / 6}}{3\left(c_{1}+c_{2} U^{1 / 6}\right)} \leq \frac{1}{3} \\
\frac{\alpha_{R}^{\prime}(U)\left(U-T_{a}\right)}{\alpha_{R}(U)}=\frac{3 \tilde{U}^{3}-\tilde{U}^{2} \tilde{T}_{a}-\tilde{U} \tilde{T}_{a}^{2}-\tilde{T}_{a}^{3}}{\tilde{U}^{3}+\tilde{U}^{2} \tilde{T}_{a}+\tilde{U} \tilde{T}_{a}^{2}+\tilde{T}_{a}^{3}}=\frac{6 q+8 q^{2}+3 q^{3}}{4+6 q+4 q^{2}+q^{3}} .
\end{aligned}
$$

Typical values of $q$ for considered applications are $q \leq 0.15$, then

$$
\alpha_{R}^{\prime}(U)\left(U-T_{a}\right) / \alpha_{R}(U) \leq 0.22 .
$$




\section{Computational Experiments}

In this section, we present results of numerical experiments.

We have solved problem $(2.1)-(2.3)$ on the domain $D=(0,0.05) \times(0,0.006)$, when the thickness of the upper and the lower sides of the isolator are equal to 0.003 and in the domain $D=(0,0.05) \times(0,0.009)$, when the thickness of the lower isolator is equal to 0.006 . The metal domain is defined as $D_{M}=$ $(0,0.035) \times\left(-3 \cdot 10^{-5}, 3 \cdot 10^{-5}\right)$. We use a uniform grid in the $x_{1}$ direction and a non-uniform grid in the $x_{2}$ direction. Such a grid is constructed in the following way: it is uniform in the metal domain $D_{M}$ and grid steps are increased continuously outside of this domain, e.g. for the upper isolator as

$$
h_{2 j} \sim \exp \left(\frac{\alpha\left(x_{2 j}-0.5\left(L_{2}+L_{2 M}\right)\right)}{L_{2}-0.5 L_{2 M}}\right) h_{2 M}, \quad \alpha \geq 1,
$$

where $h_{2 M}$ defines a grid step in the metal domain.

We choose the following values of coefficients: the thermal conductivity coefficient for a metal layer $K_{M}=58.5 \mathrm{~W} / \mathrm{mK}$ and for $\mathrm{ABC}$ isolator $K_{I}=$ $0.17 \mathrm{~W} / \mathrm{mK}$, the emissivity coefficient $\varepsilon=0.93$, the specific resistivity of the conductor $\rho_{0}=6.85$ and $c=0.0067$.

The obtained nonlinear finite volume scheme was linearized using the Picard method (3.9). An aggregation-based algebraic multigrid solver AGMG, developed by Y. Notay was used to solve systems of linear equations [13, 14]. Algebraic multigrid (AMG) is one of the most effective iterative methods for the solution of large, sparse linear systems obtained from the discretization of elliptic self-adjoint PDEs (see [1, 17], where results of many computational experiments with different AMG solvers are presented for diffusion, diffusionconvection, the Stokes and the Boussinesq problems in 2D and 3D domains).

Table 1. The results of application of AGMG with FGC iterative algorithm for an elliptic problem with strongly discontinuous coefficients

\begin{tabular}{ccccc}
\hline & $300 \times 384$ & $600 \times 768$ & $1200 \times 1576$ & $1800 \times 2304$ \\
\hline Numb. Iter. & 81 & 106 & 118 & 114 \\
CPU Time & 3.8 & 22 & 98 & 226 \\
\hline
\end{tabular}

AGMG was used as a preconditioner for Krylov subspace methods, e.g. PCG, GMRES algorithms ( for similar simulations of electrical cables we have used also a preconditioned version of BiCGStab $[5,6])$. The matrix of a linear system obtained after the Picard linearization is an M-matrix. Since we have the Robin type boundary conditions, this matrix is also symmetric, therefore the FCG iterative algorithm can be used as a Krylov subspace method. Even though coefficients of the elliptic problem are strongly discontinuous, the direct application of the AGMG solver was very robust (see results in Table 1, where the total number of iterations and CPU time are presented for 5 Picard's iterations). 
But a proper tuning of some parameters of the solver was required in the case when GMRES was used as a Krylov subspace method. The properties of the application should be taken in account. In order to guarantee the convergence of AMG-preconditioned Krylov solver, we have done the following modifications:

- the number of Gauss-Seidel pre-smooting and post-smoothing iterations was increased till 2 iterations;

- the number of recursion steps was limited to 3-4 steps (depending on the size of the discrete problem), therefore the size of the problem on the coarse grid remained quite large.

We note, that the robustness of MG solvers can be increased also by selecting a proper smoother, which is tailored for a given application, see [4].

Fig. 2(a) illustrates the distribution of the temperature in the case of a symmetrical composite structure and Fig. 2(b) gives results in the case when the lower part of the isolator is twice larger than the upper side (here the computational domain is defined as $D=(0,0.05) \times(0,0.009))$. In both experiments the current was equal to $I=65 \mathrm{~A}$, the external temperature $T_{a}=25 \mathrm{C}$.

In both cases the accuracy 0.001 of the Picard iterations was achieved after 7 iterations.

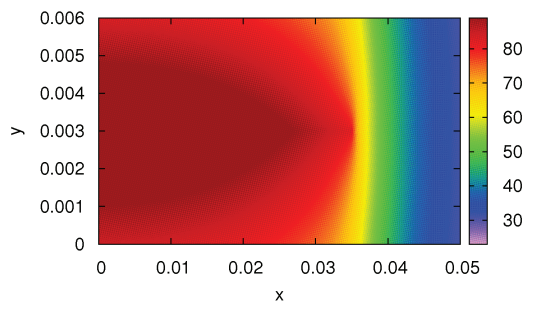

a)

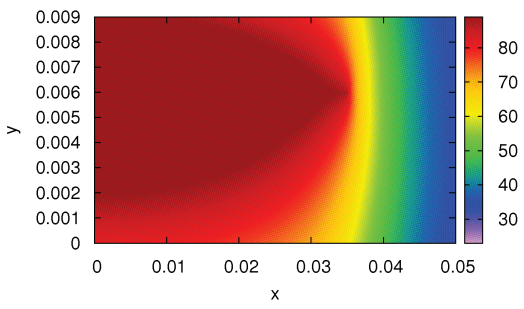

b)

Figure 2. Distribution of the temperature in the composite structure for $I=65 \mathrm{~A}$ : a) the symmetrical position of the metal layer, b) non-symmetrical position of the metal layer.

The presented numerical results illustrate our theoretical results and show the efficiency of the Picard iterative method for such applications. An open problem is a proper selection and optimization of parameters within the AMG solver, which is used to solve linearized systems of finite volume scheme. In this paper we have restricted to such values of parameters and smoothing strategies which give a robust algorithm.

\section{References}

[1] J. Boyle, J. Mihajlovic and J. Scott. HSL_MI20: An efficient AMG preconditioner for finite element problems in 3D. Int. J. Numer. Math. Engng., 2009. Doi:10.1002/nme.2758. (Published online 24 August 2009) 
[2] K. Bryan and L. Caudill. An inverse problem in thermal imaging. SIAM J. Appl. Math., 59:715-735, 1996. Doi:10.1137/S0036139994277828.

[3] P. Ciarlet, M. Schultz and R. Varga. Numerical methods of high-order accuracy for nonlinear baoundary value problems. Numerische Mathematik, 11:331-345, 1968. Doi:10.1007/BF02166686.

[4] R. Čiegis, F. Gaspar and C. Rodrigo. On parallel multiblock geometric multigrid algorithm. Computational Methods in Applied Mathematics, 8(3):223-236, 2008.

[5] R. Čiegis, A. Ilgevičius, H. Liess, M. Meilūnas and O. Suboč. Numerical simulation of the heat conduction in electrical cables. Math. Model. Anal., 12(4):425439, 2007. Doi:10.3846/1392-6292.2007.12.425-439.

[6] Raim. Čiegis, Rem. Čiegis, M. Meilūnas, G. Jankevičiūtè and V. Starikovičius. Parallel numerical algorithm for optimization of electrical cables. Math. Model. Anal., 13(4):471-482, 2008.

[7] I. Ekeland and R. Temam. Convex Analysis and Variational Problems. NorthHolland, Amsterdam, New York, Oxford, 1976.

[8] R. Ewing, O. Iliev and R. Lazarov. A modified finite volume approximation of second order elliptic with discontinuous coefficients. SIAM J. Sci. Comp., 23(4):1334-13350, 2001. Doi:10.1137/S1064827599353877.

[9] X. Hu, X. Xu and W. Chen. Numerical method for the inverse heat transfer problem in composite materials with Stefan-Boltzmann conditions. Adv. Comput. Math., 2009. Doi:10.1007/s10444-009-9131-x. (Published online 30 May 2009)

[10] F. Incropera, P. DeWitt and P. David. Introduction to Heat Transfer. John Willey \& Sons, New York, 1985.

[11] G. Jankevičiūtè and R. Čiegis. Parallel numerical solver for the simulation of the heat conduction in electrical cables. In R. Čiegis, D. Henty, B. Kagstrōm and J. Žilinskas(Eds.), Parallel Linear Algebra and Optimization: Advances and Applications. Springer Optimization and Its Applications. ISBN: 978-0-387-097060, volume 27, pp. 211-216, New-York, 2009. Springer.

[12] L. Liu, M. Huang, K. Yuan and M. Křižek. Numerical approximation of a nonlinear 3D heat radiation problem. Adv. Appl. Math. Mech., 1(1):125-139, 2009.

[13] Y. Notay. An aggregation-based algebraic multigrid method. Electronic Transactions on Numerical Analysis, 2009. (accepted for publication)

[14] Y. Notay and P. Vassilevski. Recursive krylov-based multigrid cycles. Numer. Lin. Alg. Appl., 15:473-487, 2008.

[15] Y. Saad. Iterative Methods for Sparse Linear Systems. SIAM, Philadelphia, PA, 2003.

[16] A.A. Samarskii. The Theory of Difference Schemes. Marcel Dekker, Inc., New York-Basel, 2001.

[17] K. Stüben. A review of algebraic multigrid. J. Comput. and Appl. Math., 128:281-309, 2001. Doi:10.1016/S0377-0427(00)00516-1.

[18] G. Yang, M. Yamamoto and J. Cheng. Heat transfer in composite materials with Stefan-Boltzmann interface conditions. Math. Methods Appl. Sci., 31:1297-1314, 2007. 